\title{
A Comparative Study of the Respiratory Neonatal Outcomes Utilizing Dexamethasone Sodium Phosphate versus a Mixture of Betamethasone Dipropionate and Betamethasone Sodium Phosphate as an Antenatal Corticosteroid Therapy
}

\author{
Omar F Altal' \\ Ahmed H Al Sharie $\mathbb{D}^{2}$ \\ Yazan O Al Zu'bi ${ }^{2}$ \\ Saif Aldin Rawabdeh ${ }^{3}$ \\ Wasim Khasawneh ${ }^{3}$ \\ Tamara Dawaymeh ${ }^{2}$ \\ Haneen Tashtoush ${ }^{2}$ \\ Rawan Obeidat' \\ Omar M Halalsheh ${ }^{4}$ \\ 'Department of Obstetrics \& \\ Gynecology, Faculty of Medicine, Jordan \\ University of Science \& Technology, Irbid, \\ Jordan; ${ }^{2}$ Faculty of Medicine, Jordan \\ University of Science \& Technology, Irbid, \\ Jordan; ${ }^{3}$ Department of Pediatrics and \\ Neonatology, Faculty of Medicine, Jordan \\ University of Science and Technology, \\ Irbid, Jordan; ${ }^{4}$ Department of General \\ Surgery and Urology, Faculty of Medicine, \\ Jordan University of Science \& \\ Technology, Irbid, Jordan
}

Correspondence: Omar F Altal

Department of Obstetrics \& Gynecology, Faculty of Medicine, Jordan University of Science \& Technology, P. O. Box: 3030 ,

Irbid, 221 I0, Jordan

Tel +962797423400

Fax +962 27201064

Email altal_omar@yahoo.com
Objective: The aim of this study is to compare the respiratory neonatal outcomes utilizing antenatal dexamethasone sodium phosphate (DSP) versus a mixture of betamethasone dipropionate and betamethasone sodium phosphate (B-DP/SP) for preterm births.

Patients and Methods: All neonatal intensive care unit (NICU) admissions for prematurity were retrospectively identified at our center in the period between September 2016 and September 2018. Pregnant women expected to give preterm birth and received steroid injections whether it is DSP or B-DP/SP were included in the study. Maternal and obstetrical data along with the corresponding respiratory neonatal outcomes were extracted and analyzed. The population was categorized according to the gestational age into extremely preterm (less than 28 weeks), very preterm (28 up to 32 weeks) and moderate or late preterm (32 up to 37 weeks) in which the repository outcomes were compared in each sub-group.

Results: A total of 650 premature neonates were included in the analysis. B-DP/SP illustrated a significant reduction in the occurrence of respiratory distress syndrome (RDS) among moderate or late preterm neonates $(P=0.003)$ compared to DSP. In contrast, a nonsignificant difference was observed between B-DP/SP and DSP regarding apnea of prematurity and transient tachypnea of the newborn. The number of neonates developed chronic lung disease has been remarkably reduced when using DSP in extremely $(P=0.038)$ and very $(P=0.046)$ preterm neonates when compared to B-DP/SP.

Conclusion: The dual acting B-DP/SP formulation could possess a significant potential in reducing RDS in moderate or late preterm neonates, while DSP groups exhibit a favorable result in the development of chronic lung disease in extreme and very preterm cohorts. Such findings emphasize the need of further clinical trials, pharmacokinetics, pharmacodynamics and cost effectiveness studies to evaluate the durability of these findings.

Keywords: antenatal corticosteroids, dexamethasone, betamethasone dipropionate, respiratory distress syndrome, apnea of prematurity

\section{Introduction}

Preterm birth is a fundamental concern to the standards of any health-care system with approximately 14.84 million worldwide live premature births in $2014 .^{1}$ According to the World Health Organization (WHO), premature births are defined as all births prior to 37 weeks of gestation, or less than 259 days since the first day 
of a woman's last menstrual period. ${ }^{2}$ Broadly, the causes of prematurity can be classified into two major categories comprising labor induction or caesarean births and spontaneous preterm births. The latter being further divided into two subcategories the first with intact membranes whereas the second with ruptured ones. ${ }^{3}$ Preterm birth, the world's leading cause of death in children younger than five years of age in 2016 with almost $16 \%$ of all deaths, and $35 \%$ mortality rate among newborns, presents a key obstacle to many health-care systems. ${ }^{4}$ Notwithstanding the multiple and diverse short- and longterm complications prematurity results in, among these are respiratory distress syndrome (RDS), bronchopulmonary dysplasia with other infectious and non-infectious respiratory pathologies, necrotizing enterocolitis along with retinopathy and hypoxic ischemic encephalopathy. ${ }^{5-8}$ Despite the challenging medical consequences, preterm births impose an immense psychological distress and economic burden as well. ${ }^{9,10}$

Multiple pharmacological interventions have been used for prematurity, one of which is corticosteroids, that has shown to enhance preterm management, ultimately improving morbidity and mortality rates. ${ }^{11}$ Corticosteroids can be administered either antenatally or postnatally, with a major effect on mitigating the adverse events of RDS, along with reducing the occurrence of persistent patent ductus arteriosus (PDA) and pneumonia disregarding any severe side effects such as neurodevelopmental impairment. ${ }^{12}$ The findings are attributable to its physiological and biochemical role in improving the respiratory function by replacing the natural activation of the hypothalamic-adrenal axis enhancing lung maturity, subsequently increasing the alveolar surfactant with reinforced response to surfactant therapy, along with lung compliance and total volume. Moreover, on the biochemical level, steroids induce their effects on a diversity of cellular interactions within type II pneumocytes across a range of glucocorticoid-regulated proteins in the fetal lung. ${ }^{13,14}$

Multiple guidelines were designed and implemented regarding the antenatal administration of antenatal corticosteroids including the NICE guideline, WHO recommendations, ACOG committee opinion and SOGC clinical practice guideline. ${ }^{15}$ Since both dexamethasone and betamethasone are two corticosteroids utilized as an antenatal therapy in preterm deliveries. Many studies compare the efficacy of dexamethasone sodium phosphate (DSP) versus a dual acting betamethasone formulation consisting of betamethasone sodium phosphate and betamethasone acetate. This work aims in determining the possible differences in respiratory outcomes between the use of antenatal dexamethasone versus a dual acting betamethasone formulation comprising betamethasone dipropionate and betamethasone sodium phosphate (B-DP/SP).

\section{Patients and Methods}

This retrospective single-center study was conducted at King Abdullah University Hospital (KAUH), a tertiary academic care center that is affiliated with the Jordan University of Science and Technology (JUST), to evaluate and compare the effectiveness of two types of steroidal injections; B-DP/SP versus DSP on the development of respiratory disorders of premature neonates. After obtaining the Institutional Review Board approval (IRB, Number: 2019/663), we retrospectively identified all neonatal intensive care unit (NICU) admissions for prematurity at KAUH in the period between September 2016 and September 2018. Mothers of the admitted preterm neonates were also identified. The following maternal and obstetrical information were obtained: maternal age, number of gestations, gestational age of delivery, type of steroid injection, type of delivery (either elective or emergency), mode of delivery, type of anesthesia and analgesia, maternal co-morbidities and obstetrical complications. In addition, the following neonatal data were allocated: gender, birth weight, birth depression, Apgar score at 5 minutes, RDS, transient tachypnea of newborn (TTN), meconium aspiration, apnea of prematurity, pulmonary hemorrhage, pneumothorax, pulmonary hypertension, congenital pneumonia, chronic lung disease, number of surfactant doses given, number of neonates small or large for gestational age, days of continuous mandatory ventilation (CMV) use, days of continuous positive airway pressure (CPAP) use, days of high flow nasal cannula (HFNC) use, days on room air, aminophylline use, inhaled steroid use, dexamethasone: a randomized trial (DART) regimen utilization, survival rate and length of hospitalization. Data collection was conducted by well-trained pediatric residents and interns.

Women with a singleton or multiple pregnancies expected to give preterm birth (before 37 weeks) as a result of either spontaneous preterm labor, preterm premature rupture of membranes or elective preterm birth and received steroid injections were included in the study. All alive preterm neonates admitted to the NICU, including 
inborn and out-born neonates were also included. Exclusion criteria included all stillbirths and infants who were transferred outside of our institution (due to lack of data) along with women who did not receive steroidal injections. Data were divided according to the gestational age into three groups; extremely preterm (less than 28 weeks), very preterm ( 28 up to 32 weeks) and late preterm (32 up to 37 weeks) to minimize the heterogenicity and to evaluate the effect of each steroidal formulation on the respiratory outcomes in each category (DSP vs B-DP/ SP). ${ }^{2,16}$

The obstetric service is conducted by ten full-time consultant obstetricians who follow the same guidelines. High-risk pregnancies are mostly followed by three qualified maternal-fetal medicine specialists. The gestational age of our participants was determined as documented in the electronic charts. Most of the pregnant women included in our analysis had their booking visit early in gestation and the gestational age is estimated based on early ultrasonography (US) and/or last menstrual period. Two types of steroids were utilized in our center: DSP and $\mathrm{B}-\mathrm{DP} / \mathrm{SP}$. The DSP is given twice as $12 \mathrm{mg}$ injection, 12 hours apart. B-DP/SP is formulated as a $2 \mathrm{~mL}$-sterile aqueous injectable suspension of betamethasone dipropionate and betamethasone sodium phosphate equivalent to 5 and $2 \mathrm{mg}$ betamethasone, respectively, in a sterile buffered and preserved vehicle. It is given twice, 24 hours apart.

Our NICU unit is managed by three neonatology consultants. This 30-incubator unit accommodates all inborn neonates that need specialized care. In addition, 5-10\% of the admissions are transferred from local public and private hospitals. Apart from pediatric cardiothoracic surgeries, all pediatric and surgical subspecialties are available for consultations in our center. Among the indications for NICU admission are all preterm deliveries, respiratory distress requiring any respiratory support beyond transitioning, cases that require intravenous antibiotics based on risk assessment for sepsis, and cases with major dysmorphism or suspected surgical issues. Also, newborns in the well-baby nursery who require escalation of support due to any change in their clinical exam, respiratory status, feeding issues or jaundice are transferred to the NICU. Definitions of neonatal diseases and outcomes are based on clinical presentation and supportive laboratory investigations utilizing the standard international criteria as applicable. The term prematurity was used to describe live-born neonates delivered before completing 37 weeks of gestation. RDS diagnosis was given to any preterm or occasionally term babies with signs of respiratory distress and typical chest X-ray findings of hyaline membrane disease including reticulogranular shadows, air bronchograms or white-out lung fields. Pneumothorax diagnosis was established with typical radiological findings or with positive transillumination sign. Pulmonary hypertension refers to typical echocardiographic findings using the calculated tricuspid regurgitation (TR) jet as a marker. Apnea of prematurity is a cessation of breathing for 20 seconds or longer or a shorter pause accompanied by bradycardia (less than 100 beats per minute), and cyanosis or pallor. Birth depression refers to neonates born flaccid with respiratory depression that requires resuscitation beyond the routine newborn care steps. For certain variables and outcomes, we calculated the total number of days while using a specific intervention. This was applied for CMV, CPAP, and HFNC. Number of surfactant doses are used as an indicator for the severity of neonate's condition. Surfactant is used for intubated infants on $30 \%$ or more oxygen whose clinical presentation and chest X-ray are consistent with RDS. Also, the use of aminophylline for apnea of prematurity, or the use of inhaled steroids and DART regimen for chronic lung disease are marked as severity indicators. All neonatologists follow the same institutional guidelines.

Statistical analysis has been performed as previously described. ${ }^{17}$ In brief, statistical analysis was performed using IBM SPSS statistical package for windows version 26 (Armonk, NY, USA). Data are represented as Mean \pm Standard deviation of the mean (SD) for continuous normally distributed data, median (interquartile range (IQR)) for nonnormally distributed data and frequency (percentage) for nominal data. Normality was tested using the KolmogorovSmirnov test. Chi-square test, Fisher's exact test and likelihood ratio were performed to identify any statistical significance between groups accordingly for aforementioned categorical variables and independent $t$-test for normally distributed continuous variables. $P \leq 0.05$ was considered statistically significant. Two-way analysis of variance (ANOVA) was used to compare the main effects of steroidal formulation, and gestational age on Apgar score at 5 minutes, days on CMV, days on CPAP, days on HFNC, and length of stay. Regarding two-way ANOVA, a $P \leq 0.001$ was arbitrarily considered statistically significant due to the unequal sample sizes. Partial Eta squared values were interpreted as having a small effect for $\eta^{2}>0.01$, as a medium effect for $\eta^{2}>0.06$, and as a large effect for $\eta^{2}>0.14 .^{18}$ Simple effects tests were 
performed to investigate the nature of the interaction comparing the effects of the type of steroid used within the gestational age subgroups. Regression models were used to test for the effect of any confounding variables in significantly different variables retrieved within the study groups, primarily moderate or late preterm. However, it was not performed for extremely preterm and very preterm groups due to the lack of sufficient number of observations. Binary logistic regression was used to develop a model for the dichotomous variables; RDS, meconium aspiration and inhaled steroids. If the patient developed RDS or meconium aspiration or has been given inhaled steroids a binary value of " 1 " was given otherwise a value of " 0 " was given respectively. Variables used in the models include maternal and fetal demographic characteristics; maternal age, neonatal gender, number of gestations, birth weight, mode of delivery, urgency status of the surgery, type of anesthesia used along with the maternal comorbidities.

\section{Results}

\section{Maternal and Neonatal Demographic Characteristics}

A total of 650 premature neonates admitted to the NICU were included in the analysis. The distribution of infant's gender is almost equal (males: 307 (50.3\%) and females: $303(49.7 \%))$. Singleton pregnancy was achieved in about half of the cases $(347(55.3 \%))$. The mean age of mothers at delivery was 30.5 years corresponds to a median gestational age of delivery of 34.2 weeks. Neonates were further classified according to the gestational age into extremely preterm $(24(3.7 \%))$, very preterm $(89(13.7 \%))$ and moderate or late preterm $(537(82.6 \%))$. B-DP/SP was given to 165 mothers resembling $25.4 \%$ while DSP was given to 485 reassembling $74.6 \%$ of the cases. Most deliveries were urgent in nature $(426(79.8 \%))$ in which $83.2 \%$ were cesarean sections. Spinal anesthesia was used in the majority of cases (309 (62.7\%)) compared to general anesthesia (184 (37.3)). Table 1 shows the maternal and neonatal demographic characteristics in the selected population categorized based on the type of steroid utilized. Forty-two $(6.5 \%)$ of the cases developed pre-eclampsia while only $25(3.8 \%)$ cases experienced gestational diabetes. Preterm premature rupture of membranes (PPROM) was observed in 184 $(28.4 \%)$ cases. Other maternal comorbidities were included as: chronic hypertension $(2.2 \%)$, gestational hypertension $(2.2 \%)$, diabetes mellitus (1.8\%), hypothyroidism $(2.6 \%)$, thrombophilia $(2.5 \%)$, abruption placenta $(5.1 \%)$, placenta previa $(2.2 \%)$, polyhydramnios $(0.6 \%)$, oligohydramnios
$(1.7 \%)$, and chorioamnionitis $(2.2 \%)$. Regarding maternal complication, a non-significant difference was observed in all the previously mentioned complications except for gestational diabetes $(P=0.008)$.

\section{B-DP/SP versus DSP as Antenatal Corticosteroids in Extremely Preterm Births}

A total of 24 neonates were categorized as extremely preterm with a gestational age of less than 28 weeks, which were further classified into B-DP/SP: 7 (4.2\%) and DSP: $17 \quad(3.5 \%)$ sub-groups. Table S1 shows a comparative analysis result of the respiratory neonatal outcomes and therapeutic interventions between DSP and B-DP/SP in extremely preterm neonates. DSP illustrated a significant reduction in the occurrence of chronic lung disease $(P=0.038)$. On the other hand, B-DP/SP group used assisted respiratory machines more compared to DSP as in CMV $(P<0.001)$, CPAP $(P<0.001)$ simultaneously with higher hospitalization period $(P<0.001)$. These findings are associated with a non-significant difference between B-DP/SP and DSP with regard to RDS, apnea of prematurity, transient tachypnea of the newborn, pneumothorax, pulmonary hemorrhage, birthweight, birth depression, Apgar scores, number of surfactant doses, usage of DART and aminophylline, and the number of neonates smaller for gestational age between B-DP/SP and DSP. Unfortunately, the sample size in this subgroup is extremely small impairing the statistical judgment especially in the mortality rate which need further studies to confirm these results.

\section{DSP versus B-DP/SP as Antenatal Corticosteroids in Very Preterm Births}

A total of 89 neonates were categorized as very preterm with a gestational age of 28 up to 32 weeks, which were further classified into B-DP/SP: 16 (9.7\%) and DSP: 73 (15.1\%) sub-groups. Table 2 shows a comparative analysis result of the respiratory neonatal outcomes and therapeutic interventions between DSP and B-DP/SP in very preterm neonates. The results obtained within this subgroup deviated from the trend seen in the extremely preterm group in which DSP neonates developed more chronic lung disease $(P=0.046)$ and longer duration of CPAP use $(P<0.001)$ in concomitance with nonremarkable difference in hospitalization period compared to B-DP/SP. Other previously mentioned respiratory 
Table I Maternal and Neonatal Demographic Characteristics

\begin{tabular}{|c|c|c|c|c|}
\hline Variables & Total $(n=650)$ & B-DP/SP $(n=165)$ & DSP $(n=485)$ & $P$-value \\
\hline Maternal age & $30.5 \pm 5.6$ & $30.9 \pm 5.7$ & $30.3 \pm 5.6$ & 0.264 \\
\hline Gestational age (Mean, IQR ${ }^{\mathrm{a}}$ ) & $34.2(2.8)$ & $34.5(2.5)$ & $34.2(2.9)$ & 0.161 \\
\hline Extremely preterm & $24(3.7)$ & $7(4.2)$ & $17(3.5)$ & \\
\hline Very preterm & $89(13.7)$ & $16(9.7)$ & $73(15.1)$ & \\
\hline Moderate or late preterm & $537(82.6)$ & $142(86.1)$ & $395(8 I .4)$ & \\
\hline Neonatal gender & & & & 0.162 \\
\hline Males & $307(50.3)$ & $85(55.2)$ & $222(48.7)$ & \\
\hline Females & $303(49.7)$ & $69(44.8)$ & $234(51.3)$ & \\
\hline Number of gestations & & & & 0.003 \\
\hline Singleton & $347(55.3)$ & $96(60.4)$ & $25 I(53.6)$ & \\
\hline Twins & $196(31.3)$ & $42(26.4)$ & $154(32.9)$ & \\
\hline Triplets & $72(11.5)$ & $13(8.2)$ & $59(12.6)$ & \\
\hline Quadruplets & $12(1.9)$ & $8(5.0)$ & $4(0.9)$ & \\
\hline Birth weight in grams & $2049.7 \pm 572.0$ & $2053.0 \pm 565.9$ & $2048.6 \pm 574.7$ & 0.085 \\
\hline Mode of delivery & & & & 0.422 \\
\hline Cesarean section & $54 \mid(83.2)$ & $134(8 \mid .2)$ & 407 (83.9) & \\
\hline Vaginal delivery & $109(16.8)$ & $31(18.8)$ & $78(16.1)$ & \\
\hline Elective vs Emergency & & & & 0.040 \\
\hline Elective & $108(20.2)$ & $19(14.1)$ & $89(22.3)$ & \\
\hline Emergency & $426(79.8)$ & $116(85.9)$ & $310(77.7)$ & \\
\hline Anesthesia in cesarean section & & & & 0.291 \\
\hline General anesthesia & $184(37.3)$ & $4 \mid(33.3)$ & $143(38.6)$ & \\
\hline Spinal anesthesia & $309(62.7)$ & $82(66.7)$ & $227(6 \mid .4)$ & \\
\hline Anesthesia in vaginal delivery & & & & 1.000 \\
\hline Epidural & $20(58.8)$ & $5(62.5)$ & I5 (57.7) & \\
\hline Morphine & $14(4 \mid .2)$ & $3(37.5)$ & II (42.3) & \\
\hline \multicolumn{5}{|l|}{ Maternal comorbidities } \\
\hline Chronic hypertension & $14(2.2)$ & $3(1.8)$ & II (2.3) & 1.000 \\
\hline Gestational hypertension & $14(2.2)$ & $7(4.2)$ & $7(1.4)$ & 0.055 \\
\hline Pre-eclampsia & $42(6.5)$ & $13(7.9)$ & $29(6.0)$ & 0.391 \\
\hline Gestational diabetes & $25(3.8)$ & $12(7.3)$ & $13(2.7)$ & 0.008 \\
\hline Diabetes mellitus & $12(1.8)$ & $2(1.2)$ & $10(2.1)$ & 0.740 \\
\hline Hypothyroidism & $17(2.6)$ & $3(1.8)$ & $14(2.9)$ & 0.581 \\
\hline Thrombophilia & $16(2.5)$ & $6(3.6)$ & $10(2.1)$ & 0.255 \\
\hline Abruptio placenta & $33(5.1)$ & $8(4.8)$ & $25(5.2)$ & 0.877 \\
\hline Placenta previa & $14(2.2)$ & $3(1.8)$ & II (2.3) & 1.000 \\
\hline Polyhydramnios & $4(0.6)$ & $\mathrm{I}(0.6)$ & $3(0.6)$ & 1.000 \\
\hline Oligohydramnios & II (I.7) & $5(3.0)$ & $6(1.2)$ & 0.158 \\
\hline Maternal $\mathrm{HBsAg}^{\mathrm{b}}$ & $5(0.8)$ & I (0.6) & $4(0.8)$ & 1.000 \\
\hline Premature rupture of membranes & $184(28.4)$ & $46(27.9)$ & $138(28.6)$ & 0.853 \\
\hline Chorioamnionitis & $14(2.2)$ & $6(3.6)$ & $8(1.7)$ & 0.210 \\
\hline
\end{tabular}

Notes: Data are presented as mean \pm standard deviation (SD) or frequency (percentage). ${ }^{\mathrm{a}}$ Interquartile range. ${ }^{\mathrm{b}}$ Reactive Hepatitis $\mathrm{B}$ surface antigen.

outcomes including RDS, apnea of prematurity, pneumothorax, pulmonary hemorrhage, meconium aspiration, birthweight, birth depression, Apgar scores, number of surfactant doses, usage of DART and aminophylline, and the number of neonates smaller and larger for gestational age were totally non-significant between B-DP/SP and DSP groups.

\section{DSP versus B-DP/SP as Antenatal Corticosteroids in Moderate or Late Preterm Births}

A total of 537 neonates were categorized as moderate or late preterm with a gestational age of 32 up to 37 weeks, which were further classified into B-DP/SP: 142 (86.1\%) and DSP: 395 (81.4\%) sub-groups. Table 3 shows a comparative 
Table 2 A Comparative Analysis of the Respiratory Neonatal Outcomes and Therapeutic Interventions Between DSP and B-DP/SP in Very Preterm Neonates

\begin{tabular}{|c|c|c|c|}
\hline \multirow[t]{2}{*}{ Variables } & \multicolumn{3}{|c|}{ Very Preterm $(n=89)$} \\
\hline & B-DP/SP $(n=16)$ & $\operatorname{DSP}(n=73)$ & $P$-value \\
\hline Respiratory distress syndrome & $13(81.3)$ & $68(93.2)$ & 0.151 \\
\hline Apnea of prematurity & I (6.3) & $17(23.3)$ & 0.176 \\
\hline Transient tachypnea of the newborn & $0(0.0)$ & $0(0.0)$ & $*$ \\
\hline Chronic lung disease & & & 0.046 \\
\hline No & $15(93.8)$ & $50(69.4)$ & \\
\hline Mild & I (6.3) & $12(16.7)$ & \\
\hline Moderate & $0(0.0)$ & $10(13.9)$ & \\
\hline Severe & $0(0.0)$ & $0(0.0)$ & \\
\hline Pneumothorax & I (6.3) & $3(4.2)$ & 0.563 \\
\hline Congenital pneumonia & $0(0.0)$ & $0(0.0)$ & $*$ \\
\hline Pulmonary hemorrhage & $0(0.0)$ & I (I.4) & 1.000 \\
\hline Pulmonary hypertension & $0(0.0)$ & $0(0.0)$ & $*$ \\
\hline Meconium aspiration & $0(0.0)$ & I (3.3) & 1.000 \\
\hline Birth weight in grams & $1336.9 \pm 425.5$ & $1419.5 \pm 324.5$ & 0.499 \\
\hline Birth Depression & $0(0.0)$ & $7(9.6)$ & 0.343 \\
\hline Small for gestational age & $8(50.0)$ & $24(32.9)$ & 0.196 \\
\hline Large for gestational age & $0(0.0)$ & I (I.4) & 1.000 \\
\hline Apgar score at 5 minutes & $8.2 \pm 0.8$ & $8.0 \pm 1.4$ & 0.564 \\
\hline Number of surfactant dosages & & & 0.227 \\
\hline 0 & $7(50.0)$ & $18(24.7)$ & \\
\hline I & $3(21.4)$ & $33(45.2)$ & \\
\hline 2 & $3(21.4)$ & $14(19.2)$ & \\
\hline 3 & $I(7.1)$ & $8(11.0)$ & \\
\hline 4 & $0(0.0)$ & $0(0.0)$ & \\
\hline Use of aminophylline & $8(50.0)$ & $47(64.4)$ & 0.284 \\
\hline Inhaled steroids & $6(37.5)$ & $35(47.9)$ & 0.448 \\
\hline DART ${ }^{a}$ & $\mathrm{I}(6.3)$ & $9(12.3)$ & 0.683 \\
\hline Days on $\mathrm{CMV}^{\mathrm{b}}$ & $1.9 \pm 3.0$ & $5.7 \pm 8.2$ & 0.022 \\
\hline Days on CPAPC & $7.6 \pm 5.7$ & $14.9 \pm 12.1$ & $<0.001$ \\
\hline Days on $\mathrm{HFNC}^{\mathrm{d}}$ & $0.0 \pm 0.0$ & $1.9 \pm 5.7$ & 0.33 \\
\hline Length of stay in days & $28.1 \pm 10.5$ & $34.8 \pm 21.4$ & 0.059 \\
\hline Mortality (Alive percentage) & $15(93.8)$ & $63(86.3)$ & 0.681 \\
\hline
\end{tabular}

Notes: Data are presented as mean \pm standard deviation (SD) or frequency (percentage) within B-DP/SP and DSP groups. ${ }^{*}$ Cannot generate a $P$-value due to insufficient data. ${ }^{a}$ Dexamethasone: A Randomized Trial. ${ }^{b}$ Continuous mandatory ventilation. ${ }^{c}$ Continuous positive airway pressure. ${ }^{d} \mathrm{High}$ flow nasal cannula.

analysis result of the respiratory neonatal outcomes and therapeutic interventions between DSP and B-DP/SP in moderate or late preterm neonates. B-DP/SP group showed a significant lower rate of $\operatorname{RDS}(P=0.003)$ compared to DSP with non-significant difference in apnea of prematurity, transient tachypnea of the newborn, pneumothorax, pulmonary hemorrhage, birthweight, birth depression, Apgar scores, number of surfactant doses, and the number of neonates smaller and larger for gestational age between B-DP/SP and DSP except for meconium aspiration $(P=0.046)$. B-DP/SP group showed a lower usage for inhaled steroids $(P=0.014)$ compared to DSP with nonsignificant difference in the usage of aminophylline, DART, CMV, CPAP, and
HFNC associated with unremarkable difference in hospitalization period and mortality rates.

\section{Logistic Regression Models}

A binary logistic regression was performed to ascertain the effects of maternal and fetal demographic characteristics and maternal comorbidities on the likelihood for developing RDS in moderate or late preterm pregnancies. The logistic regression model was statistically significant, $\chi^{2}(26)=116.573, P<$ 0.001 . The model explained $28.7 \%$ (Nagelkerke $\mathrm{R}^{2}$ ) of the variance in RDS and correctly classified $68.2 \%$ of the cases. Neonatal gender $(P=0.045)$, number of gestations $(P=0.002)$, birth weight $(P<0.001)$, placenta previa $(P=0.016)$, 
Table 3 A Comparative Analysis of the Respiratory Neonatal Outcomes and Therapeutic Interventions Between DSP and B-DP/SP in Very Preterm Neonates

\begin{tabular}{|c|c|c|c|}
\hline \multirow[t]{2}{*}{ Variables } & \multicolumn{3}{|c|}{ Moderate or Late Preterm $(n=537)$} \\
\hline & B-DP/SP $(n=142)$ & DSP $(n=395)$ & $P$-value \\
\hline Respiratory distress syndrome & $42(29.6)$ & $172(43.9)$ & 0.003 \\
\hline Apnea of prematurity & $7(4.9)$ & $36(9.2)$ & 0.107 \\
\hline Transient tachypnea of the newborn & II (7.7) & $26(6.6)$ & 0.654 \\
\hline Chronic lung disease & & & 0.603 \\
\hline No & $140(98.6)$ & $382(98.2)$ & \\
\hline Mild & I (0.7) & $4(4.0)$ & \\
\hline Moderate & I (0.7) & $\mathrm{I}(0.3)$ & \\
\hline Severe & $0(0.0)$ & $2(0.5)$ & \\
\hline Pneumothorax & $0(0.0)$ & $2(0.5)$ & 1.000 \\
\hline Congenital pneumonia & $0(0.0)$ & $0(0.0)$ & $*$ \\
\hline Pulmonary hemorrhage & $\mathrm{I}(0.7)$ & $0(0.0)$ & 0.267 \\
\hline Pulmonary hypertension & $0(0.0)$ & $0(0.0)$ & $*$ \\
\hline Meconium aspiration & $4(7.8)$ & $2(1.4)$ & 0.046 \\
\hline Birth weight in grams & $2191.2 \pm 455.3$ & $2219.1 \pm 466.3$ & 0.519 \\
\hline Birth Depression & $3(2.1)$ & II (2.8) & 0.769 \\
\hline Small for gestational age & $43(30.3)$ & $119(30.1)$ & 0.972 \\
\hline Large for gestational age & $3(2.1)$ & $6(1.5)$ & 0.705 \\
\hline Apgar score at 5 minutes & $8.6 \pm 1.1$ & $8.6 \pm 1.0$ & 0.983 \\
\hline Number of surfactant dosages & & & 0.101 \\
\hline 0 & $72(78.3)$ & $174(70.2)$ & \\
\hline 1 & $16(17.4)$ & $58(23.4)$ & \\
\hline 2 & $3(3.3)$ & II (4.4) & \\
\hline 3 & $0(0.0)$ & $5(2.0)$ & \\
\hline 4 & $\mathrm{I}(\mathrm{I} . \mathrm{I})$ & $0(0.0)$ & \\
\hline Use of aminophylline & $\mathrm{I}(0.7)$ & $12(3.1)$ & 0.201 \\
\hline Inhaled steroids & $4(2.8)$ & $36(9.2)$ & 0.014 \\
\hline DART ${ }^{\mathrm{a}}$ & $2(1.4)$ & $4(1.0)$ & 0.659 \\
\hline Days on $\mathrm{CMV}^{\mathrm{b}}$ & $0.5 \pm 2.0$ & $0.9 \pm 3.6$ & 0.646 \\
\hline Days on CPAPC & $2.4 \pm 4.6$ & $3.8 \pm 4.9$ & 0.051 \\
\hline Days on $\mathrm{HFNC}^{\mathrm{d}}$ & $0.2 \pm 1.0$ & $0.3 \pm 1.8$ & 0.651 \\
\hline Length of stay in days & $8.7 \pm 10.8$ & $9.6 \pm 9.2$ & 0.510 \\
\hline Mortality (Alive percentage) & I 38 (97.9) & $377(96.7)$ & 0.578 \\
\hline
\end{tabular}

Notes: Data are presented as mean \pm standard deviation (SD) or frequency (percentage) within B-DP/SP and DSP groups. *Cannot generate a $P$-value due to insufficient data. ${ }^{a}$ Dexamethasone: A Randomized Trial. ${ }^{b}$ Continuous mandatory ventilation. ${ }^{c}$ Continuous positive airway pressure. ${ }^{d} \mathrm{High}$ flow nasal cannula.

premature rupture of membranes $(P=0.002)$, and maternal chorioamnionitis $(P=0.014)$, all significantly affected the development of RDS in addition to the type of steroid used $(P=0.001)$ (Table S2). The model for meconium aspiration was statistically insignificant $\chi^{2}(24)=27.399, P=0.286$. With none of the variables having any predictability role in the development of meconium aspiration for moderate or late preterm births (Table S3). Table S4 Represents binary logistic regression values with the inhaled steroids as a dependent variable; the third model concerning the requirement of inhaled steroids was statistically significant $\chi^{2}(26)=103.029, P<$ 0.001 and it was able to explain $35.3 \%$ (Nagelkerke $\mathrm{R}^{2}$ ) of the variance and could correctly classify $88.5 \%$ of the cases. Birth weight $(P<0.001)$ along with placenta previa $(P=0.012)$ and the number of gestations-quadruplets $(P=0.022)$ significantly resulted in the use of inhaled steroids. However, the type of steroid did not have any affect $(P=0.062)$ hence the significant result retrieved using Chi-square test can be the result of the confounding influence of majorly birth weight and the presence of placenta previa.

\section{Discussion}

The current study provides a novel insight to the effect of steroidal formulation on the development of neonatal 
respiratory complications. The key findings include a significant reduction in the occurrence of RDS among moderate or late preterm neonates $(P=0.003)$ compared in the B-DP/SP group compared to DSP. In contrast, a non-significant difference was observed between B-DP/ SP and DSP regarding apnea of prematurity and transient tachypnea of the newborn. The number of neonates developed chronic lung disease has been remarkably reduced when using DSP in extremely $(P=0.038)$ and very $(P=$ $0.046)$ preterm neonates when compared to B-DP/SP. No effect was observed with respect to pneumothorax, pulmonary hypertension, pulmonary hemorrhage, congenital pneumonia, meconium aspiration, birthweight, birth depression, Apgar scores and the number of neonates smaller and larger for gestational age between B-DP/SP and DSP.

Since 1972, when Liggins and Howie discovered the importance of antenatal corticosteroid therapy for preterm infants with its outstanding role in improving the mortality and morbidity rates, a large growing body of the literature has been devoted to study such rationale including which type of corticosteroids should be utilized, its dose and dosage form along with the corresponding short and longterm sequalae, which induced a lot of controversies in the medical literature. ${ }^{19,20}$ One of these arguable questions is whether to use betamethasone or dexamethasone in such setting? Crowther et al have performed a multicenter double-blind, randomized controlled trial to evaluate the mortality and neurosensory disabilities of intramuscular dexamethasone versus betamethasone in preterm infants. The study enrolled 1346 women along with 1509 fetuses which revealed a non-significant difference between both groups regarding deaths and neurosensory disabilities at the age of 2 years. ${ }^{21}$ An updated meta-analysis including 30 studies to evaluate the role of antenatal corticosteroids in the fetal lung maturation for women at risk of preterm birth. The analysis has included 7774 pregnant women and 8158 infants which revealed that antenatal corticosteroids significantly decreased perinatal death, neonatal death, RDS, intraventricular hemorrhage, necrotizing enterocolitis, need for mechanical ventilation and systemic infections during the first 48 hours of life. Adding to this, corticosteroids therapy did not impact chronic lung disease, mean birthweight, neurodevelopment delay along with childhood or adulthood mortality rates. One of the highlighted results of the same analysis suggests no definite or significant difference between betamethasone or dexamethasone in the mentioned context. ${ }^{22}$
Most of these studies compare dexamethasone in its sodium phosphate form versus a mixture of betamethasone acetate and betamethasone sodium phosphate. Herein, we represent for the first time, a comprehensive retrospective clinical analysis to evaluate the respiratory outcomes difference between DSP and an intramuscular injection composed of a mixture of betamethasone dipropionate and betamethasone sodium phosphate, as an antenatal therapy for expected preterm deliveries. B-DP/SP is considered as dual acting drug formulation that exhibits rapid and immediate short action due to the fast hydrolysis and release of betamethasone sodium phosphate while the dipropionate ester has slow hydrolysis rate leading to a sustained release effect from the site of injection. ${ }^{23}$ A similar mechanism of action is seen by replacing the dipropionate ester with acetate ester. This formulation is more popular and has been used in a variety of clinical applications and studies compared to B-DP/SP formula. ${ }^{24}$ Jobe et al have previously determined the minimal fetal therapeutic concentration of corticosteroids which is about $1 \mathrm{ng} / \mathrm{mL}$ for a continuous 48 hours using several animal models and neonatal blood samples data. The authors suggest that the long-acting betamethasone acetate is not ideal for an antenatal corticosteroid since it is associated with prolonged fetal exposure, carrying the risk of unwanted complications and side effects. ${ }^{25}$ The dual acting formulation is designed to enhance its efficacy with the administration of single injection per day. This rationale is still questionable regarding its efficacy and safety.

The current study has been performed in a retrospective manner due to the variability of corticosteroids supplied between DSP and B-DP/SP, associated with the large number of deliveries in KAUH since it is the leading tertiary hospital in northern Jordan. ${ }^{26}$ A total of 650 neonates were retrospectively identified as preterm infants with antenatal corticosteroids therapy. The selected population of neonates covers a wide spectrum of clinical settings with several numbers of gestations, modes of delivery and maternal comorbidities. Neonates in both groups have a similar mean maternal and gestational age with no significance seen. The included neonatal population have a similar trend regarding birth weight, B-DP/SP and DSP groups have a birth weight mean value of 2053.0 \pm 565.9 and $2048.6 \pm 574.7$ grams, respectively. Subsequently, a non-remarkable difference was also observed in the percentage of neonates smaller and larger for the corresponding gestational age in both groups. It has been previously reported that antenatal corticosteroids 
therapy reduces birth weight, height and head circumference without a known clinical significance in term neonates. ${ }^{27,28}$ In addition, multiple doses of antenatal corticosteroids exposure have been linked to an apparent decrease in fetal growth in a dose-response manner with the possibility of long-term developmental effects. ${ }^{29-31}$ On the other hand, the previously mentioned meta-analysis indicates that antenatal corticosteroids do not influence birthweight in preterm neonates. ${ }^{22}$

The major highlight of the current study is the significant reduction in the incidence of RDS in neonates treated with B-DP/SP compared to DSP in moderate or late preterm $(P=0.003)$ with a non-significant difference observed in extreme and very preterm. In the latest published systematic review and network meta-analysis discussing the differences between dexamethasone and betamethasone for preterm births which engaged 11,227 women and 11,878 infants throughout 45 studies. It revealed a non-significant difference regarding neonatal mortality rates, intraventricular hemorrhage, neurodevelopmental disabilities and birthweight. Dexamethasone has been associated with higher rates in developing endometritis and RDS. However, it can decrease the development of chorioamnionitis and fetal death. ${ }^{32}$ These findings could be attributed to the pharmacokinetic properties of each formulation. A 1:1 mixture of betamethasone phosphate and betamethasone acetate has a fast and sustained release in which its plasma levels could be detected after 14 days of a single intramuscular injection. Moreover, dexamethasone has a terminal half-life of 5.5 hours compared to 11 hours with the dual acting betamethasone mixture. ${ }^{33}$ From a pharmacodynamic point of view, the dual acting betamethasone formulation cause a longstanding adrenal suppression associated with high glucose levels, an increase in neutrophils and a decrease in basophils and lymphocytes counts (CD3CD4 and CD3CD8 subsets). ${ }^{33}$

The occurrence rate of TTN in the tested groups was non-significant across the gestational age categories. To the best of our knowledge, no definitive studies have explored the differences between corticosteroids subtypes and their impact in the occurrence of TTN in preterm neonates. In the current study, we could not identify preterm neonates who developed TTN without corticosteroids therapy since many preterm deliveries in KAUH are treated antenatally with DSP or B-DP/SP, so, the role of corticosteroids in the incidence of TTN could not be determined without a control group. Antenatal corticosteroids exhibit its greatest effect in reduction of TTN when given beyond 34 weeks of gestation, such intervention should be outwaited for serious side effects as neonatal hypoglycemia. ${ }^{34}$ But its role in the late preterm (34-36 weeks of gestation) is still controversial, some case control studies and randomized clinical trials indicate that antenatal corticosteroids do not reduce the incidence of respiratory disorders in newborns, neither its outcomes nor morbidities. ${ }^{35-37}$ On the other hand, several randomized clinical trials suggest that corticosteroids are beneficial in reducing respiratory complications for late preterm neonates including TTN. ${ }^{38,39}$

The occurrence rates of pneumothorax in both groups were extracted since it is a serious and life-threatening respiratory complication of RDS in preterm neonates. ${ }^{40}$ Both groups illustrated a non-remarkable statistical difference in the occurrence of pneumothorax. Overwhelming evidence supports the fact that antenatal corticosteroids therapy whether it is dexamethasone or betamethasone based does not impact pneumothorax. In a prospective study that engaged 11,022 preterm infants revealed that corticosteroids therapy does not affect pneumothorax or pulmonary hemorrhage, such findings are concurrent with the non-significant results among tested groups. ${ }^{41}$ Another prospective observational study conducted to evaluate the comorbidities among late preterm infants revealed that exposure to prenatal corticosteroids did not affect the incidence of pneumothorax. The same study suggests that corticosteroids therapy did not significantly decrease the need for CMV, CPAP or fluid therapy while the use of surfactants and oxygen therapy with NICU admission were remarkably reduced in the treated groups. ${ }^{42}$ It has been elucidated that antenatal corticosteroid whether it is betamethasone or dexamethasone based do not impact the incidence of chronic lung disease as previously described. ${ }^{22}$ In contrast, this work suggests a significant reduction in chronic lung disease among extreme and very preterm neonates.

\section{Conclusion}

The current work describes for the first time the neonatal respiratory outcomes utilizing DSP versus B-DP/SP for preterm births. Significant changes regarding the mentioned outcomes emphasize the role of a specific antenatal corticosteroid formulation with its relation to multiple short-term respiratory outcomes. The current study possesses several limitations represented by its retrospective nature. We could not identify preterm infants without antenatal corticosteroid therapy. The lack of control 
group restricts the ability to differentiate between a nonsignificant result between study groups and the role of corticosteroid in the occurrence of specific pathological setting. A limited sample size noticed in the extreme and very preterm categories could impact any generated significances which needs further evaluation. The long-term effects of B-DP/SP were not addressed considering the lack of data availability in the computerized system. Antenatal corticosteroids have been previously linked to mental and behavioral disorders in children as observed in 674,877 singletons born in Finland. ${ }^{43}$ Future work should focus on addressing non-respiratory long- and short-term effects as in the case of neurosensory disabilities and endocrinopathies. Comprehensive pharmacokinetics and pharmacodynamics studies to compare multiple dualacting betamethasone formulations are also endorsed. In addition, a cost effectiveness study for B-DP/SP versus DSP is also recommended.

\section{Data Sharing Statement}

The current project's data were obtained firstly through an automated search in the electronic medical records of KAUH followed by extensive data extraction and verification by a well-trained team of interns and residents. Data are available upon request from the corresponding author (Omar Altal) for further analysis and evaluation. No specific regulations were created or discussed in our institution regarding anonymous data sharing of individual patients, so our team decided to share it upon request rather than uploading a public-based deposit.

\section{Statement of Ethics}

The study was approved by the institutional review board committee in KAUH. This study was conducted in accordance with the declaration of Helsinki, applying all the current regulations for retrospective studies in KAUH. Patient consents were waived as data was used in aggregate anonymously.

\section{Author Contributions}

All authors made a significant contribution to the work reported, whether that is in the conception, study design, execution, acquisition of data, analysis and interpretation, or in all these areas; took part in drafting, revising or critically reviewing the article; gave final approval of the version to be published; have agreed on the journal to which the article has been submitted; and agree to be accountable for all aspects of the work.

\section{Disclosure}

The authors declare no conflicts of interest in this work.

\section{References}

1. Chawanpaiboon S, Vogel JP, Moller A-B, et al. Global, regional, and national estimates of levels of preterm birth in 2014: a systematic review and modelling analysis. Lancet Glob Health. 2019;7(1):e37e46. doi:10.1016/S2214-109X(18)30451-0

2. Dbstet A. WHO: recommended definitions, terminology and format for statistical tables related to the perinatal period and use of a new certificate for cause of perinatal deaths. Modifications recommended by FIGO as amended October 14, 1976. Acta Obstet Gynecol Scand. 1977;56(3):247-253.

3. Goldenberg RL, Culhane JF, Iams JD, Romero R. Epidemiology and causes of preterm birth. Lancet. 2008;371(9606):75-84. doi:10.1016/ S0140-6736(08)60074-4

4. You D, New J, Wardlaw T. Levels and Trends in Child Mortality. Report 2015. Estimates Developed by the UN Inter-Agency Group for Child Mortality Estimation. New York: United Nations Children's Fund; 2015:2017.

5. Platt MJ. Outcomes in preterm infants. Public Health. 2014;128 (5):399-403. doi:10.1016/j.puhe.2014.03.010

6. Kurinczuk JJ, White-Koning M, Badawi N. Epidemiology of neonatal encephalopathy and hypoxic-ischaemic encephalopathy. Early Hum Dev. 2010;86(6):329-338. doi:10.1016/j.earlhumdev.2010.05.010

7. Torloni MR, Betrán AP, Daher S, et al. Maternal BMI and preterm birth: a systematic review of the literature with meta-analysis. J Matern Fetal Neonatal Med. 2009;22(11):957-970. doi:10.3109/ 14767050903042561

8. Lin PW, Stoll BJ. Necrotising enterocolitis. Lancet. 2006;368 (9543):1271-1283. doi:10.1016/S0140-6736(06)69525-1

9. Pisoni C, Spairani S, Manzoni F, et al. Depressive symptoms and maternal psychological distress during early infancy: a pilot study in preterm as compared with term mother-infant dyads. J Affect Disord. 2019;257:470-476. doi:10.1016/j.jad.2019.07.039

10. Bérard A, Le Tiec M, De Vera MA. Study of the costs and morbidities of late-preterm birth. Arch Dis Child Fetal Neonatal Ed. 2012;97 (5):F329-F334. doi:10.1136/fetalneonatal-2011-300969

11. Travers CP, Clark RH, Spitzer AR, Das A, Garite TJ, Carlo WA. Exposure to any antenatal corticosteroids and outcomes in preterm infants by gestational age: prospective cohort study. BMJ. 2017;356: j1039-j1039. doi:10.1136/bmj.j1039

12. Delara M, Chauhan BF, Le ML, Abou-Setta AM, Zarychanski R, TJong GW. Efficacy and safety of pulmonary application of corticosteroids in preterm infants with respiratory distress syndrome: a systematic review and meta-analysis. Arch Dis Child Fetal Neonatal Ed. 2019;104(2):F137-f144. doi:10.1136/archdischild2017-314046

13. Ballard PL, Ballard RA. Scientific basis and therapeutic regimens for use of antenatal glucocorticoids. Am J Obstet Gynecol. 1995;173 (1):254-262. doi:10.1016/0002-9378(95)90210-4

14. Hallman M, Peltoniemi O, Kari MA. Enhancing functional maturity before preterm birth. Neonatology. 2010;97(4):373-378. doi:10.1159/ 000297768

15. Tsakiridis I, Mamopoulos A, Athanasiadis A, Dagklis T. Antenatal corticosteroids and magnesium sulfate for improved preterm neonatal outcomes: a review of guidelines. Obstet Gynecol Surv. 2020;75 (5):298-307. doi:10.1097/ogx.0000000000000778

16. Blencowe H, Cousens S, Oestergaard MZ, et al. National, regional, and worldwide estimates of preterm birth rates in the year 2010 with time trends since 1990 for selected countries: a systematic analysis and implications. Lancet. 2012;379(9832):2162-2172. doi:10.1016/ s0140-6736(12)60820-4 
17. Alshari O, Al Zu'bi YO, Al Sharie AH, et al. Evaluating the prognostic role of monocytopenia in chemotherapy-induced febrile neutropenia patients treated with granulocyte colony-stimulating factor. Ther Clin Risk Manag. 2021;17:963-973. doi:10.2147/TCRM. S318370

18. Guéguen N, Lourel M, Pascual A, Mouda F. A simple method to determine and evaluate "effect size". Sante. 2011;21(2):103-105. L'importance d'un effet: méthodologie simple de détermination et d'évaluation de l'« effect size ». doi:10.1684/san.2011.0240

19. Liggins GC, Howie RN. A controlled trial of antepartum glucocorticoid treatment for prevention of the respiratory distress syndrome in premature infants. Pediatrics. 1972;50(4):515.

20. Lee SK, Qi Z. Antenatal corticosteroids prior to preterm birth: betamethasone or dexamethasone? Lancet Child Adolesc Health. 2019;3 (11):750-751. doi:10.1016/S2352-4642(19)30293-7

21. Crowther CA, Ashwood P, Andersen CC, et al. Maternal intramuscular dexamethasone versus betamethasone before preterm birth (ASTEROID): a multicentre, double-blind, randomised controlled trial. Lancet Child Adolesc Health. 2019;3(11):769-780. doi:10.1016/S2352-4642(19)30292-5

22. Roberts D, Brown J, Medley N, Dalziel SR. Antenatal corticosteroids for accelerating fetal lung maturation for women at risk of preterm birth. Cochrane Database Syst Rev. 2017;3(3):CD004454CD004454. doi:10.1002/14651858.CD004454.pub3

23. Kopylov AT, Novikov AA, Kaysheva AL, et al. Quantitative assessment of betamethasone dual-acting formulation in urine of patients with rheumatoid arthritis and ankylosing spondylitis after single-dose intramuscular administration and its application to long-term pharmacokinetic study. J Pharm Biomed Anal. 2018;149:278-289. doi:10.1016/j.jpba.2017.11.021

24. Salem II, Najib NM. Pharmacokinetics of betamethasone after single-dose intramuscular administration of betamethasone phosphate and betamethasone acetate to healthy subjects. Clin Ther. 2012;34 (1):214-220. doi:10.1016/j.clinthera.2011.11.022

25. Jobe AH, Kemp M, Schmidt A, Takahashi T, Newnham J, Milad M. Antenatal corticosteroids: a reappraisal of the drug formulation and dose. Pediatr Res. 2020;2020. doi:10.1038/s41390-020-01249-w

26. Altal OF, Aleshawi AJ, Khrais MZ, et al. The practice of intracytoplasmic sperm injection in Jordan: a clinical outcome study. Ann Med Surg. 2020;57:196-200. doi:10.1016/j.amsu.2020.07.042

27. Thorp JA, Jones PG, Knox E, Clark RH. Does antenatal corticosteroid therapy affect birth weight and head circumference? Obste Gynecol. 2002;99(1):101-108. doi:10.1016/S0029-7844(01)01656-8

28. Bloom SL, Sheffield JS, McIntire DD, Leveno KJ. Antenatal dexamethasone and decreased birth weight. Obstet Gynecol. 2001;97 (4):485-490. doi:10.1016/S0029-7844(00)01206-0

29. Murphy KE, Willan AR, Hannah ME, et al. Effect of antenatal corticosteroids on fetal growth and gestational age at birth. Obstet Gynecol. 2012;119(5):917-923. doi:10.1097/AOG.0b013e31825189dc

30. Norberg H, Stålnacke J, Heijtz RD, et al. Antenatal corticosteroids for preterm birth: dose-dependent reduction in birthweight, length and head circumference. Acta Paediatr. 2011;100(3):364-369. doi:10.1111/j.1651-2227.2010.02074.x
31. French NP, Hagan R, Evans SF, Godfrey M, Newnham JP. Repeated antenatal corticosteroids: size at birth and subsequent development. Am J Obstet Gynecol. 1999;180(1):114-121. doi:10.1016/S00029378(99)70160-2

32. Ciapponi A, Klein K, Colaci D, et al. Dexamethasone vs. betamethasone for preterm birth: a systematic review and network meta-analysis. Authorea. 2020. doi:10.22541/au.159248834.46935661

33. Jobe AH, Milad MA, Peppard T, Jusko WJ. Pharmacokinetics and pharmacodynamics of intramuscular and oral betamethasone and dexamethasone in reproductive age women in India. Clin Transl Sci. 2020;13(2):391-399. doi:10.1111/cts.12724

34. Kamath-Rayne BD, Rozance PJ, Goldenberg RL, Jobe AH. Antenatal corticosteroids beyond 34 weeks gestation: what do we do now? Am J Obstet Gynecol. 2016;215(4):423-430. doi:10.1016/j.ajog.2016.06.023

35. Porto AMF, Coutinho IC, Correia JB, Amorim MMR. Effectiveness of antenatal corticosteroids in reducing respiratory disorders in late preterm infants: randomised clinical trial. BMJ. 2011;342:d1696. doi:10.1136/bmj.d1696

36. Gyamfi-Bannerman C, Gilbert S, Landon MB, et al. Effect of antenatal corticosteroids on respiratory morbidity in singletons after late-preterm birth. Obstet Gynecol. 2012;119(3):555-559. doi:10.1097/AOG.0b013e31824758f6

37. Kirshenbaum M, Mazaki-Tovi S, Amikam U, et al. Does antenatal steroids treatment prior to elective cesarean section at 34-37 weeks of gestation reduce neonatal morbidity? Evidence from a case control study. Arch Gynecol Obstet. 2018;297(1):101-107. doi:10.1007/ s00404-017-4557-8

38. Üstün N, Hocaoğlu M, Turgut A, Arslanoğlu S, Ovalı F. Does antenatal corticosteroid therapy improve neonatal outcomes in late preterm birth? J Matern Fetal Neonatal Med. 2020:1-7. doi:10.1080/ 14767058.2020 .1808614

39. Gyamfi-Bannerman C, Thom EA, Blackwell SC, et al. Antenatal betamethasone for women at risk for late preterm delivery. $N \mathrm{Engl}$ J Med. 2016;374(14):1311-1320. doi:10.1056/NEJMoa1516783

40. Ward RM, Beachy JC. Neonatal complications following preterm birth. BJOG. 2003;110(s20):8-16. doi:10.1046/j.14710528.2003.00012.x

41. Travers CP, Carlo WA, McDonald SA, et al. Mortality and pulmonary outcomes of extremely preterm infants exposed to antenatal corticosteroids. Am J Obstet Gynecol. 2018;218(1):130.e1-130.e13. doi:10.1016/j.ajog.2017.11.554

42. Gázquez Serrano IM, Arroyos Plana A, Díaz Morales O, Herráiz Perea C, Holgueras Bragado A. Antenatal corticosteroid therapy and late preterm infant morbidity and mortality. An Pediatr. 2014;81 (6):374-382. doi:10.1016/j.anpede.2014.01.003

43. Räikkönen K, Gissler M, Kajantie E. Associations between maternal antenatal corticosteroid treatment and mental and behavioral disorders in children. JAMA. 2020;323(19):1924-1933. doi:10.1001/ jama.2020.3937
International Journal of General Medicine

\section{Publish your work in this journal}

The International Journal of General Medicine is an international, peer-reviewed open-access journal that focuses on general and internal medicine, pathogenesis, epidemiology, diagnosis, monitoring and treatment protocols. The journal is characterized by the rapid reporting of reviews, original research and clinical studies
Dovepress

across all disease areas. The manuscript management system is completely online and includes a very quick and fair peer-review system, which is all easy to use. Visit http://www.dovepress.com/ testimonials.php to read real quotes from published authors. 